\title{
Rational Strain Engineering in Delafossite Oxides for Highly Efficient Hydrogen Evolution Catalysis in Acidic Media
}

Filip Podjaski ${ }^{1,2}$, Daniel Weber ${ }^{1,3}$, Siyuan Zhang ${ }^{4}$, Leo Diehl $^{1,3},{\text { Roland } \text { Eger }^{1} \text {, Viola Duppel }}^{1}$, Esther AlarconLlado $^{5}$, Gunther Richter ${ }^{6}$, Frederik Haase ${ }^{1,3}$, Anna Fontcuberta i Morral ${ }^{2,7}$, Christina Scheu ${ }^{4}$, Bettina V. Lotsch*1,3,8,9

${ }^{1}$ Max-Planck-Institute for Solid State Research, Heisenbergstraße 1, 70569 Stuttgart, Germany. ${ }^{2}$ Laboratory of Semiconductor Materials, Institute of Materials, Faculty of Engineering, Ecole Polytechnique Fédérale de Lausanne, Station 12, 1015 Lausanne, Switzerland. ${ }^{3}$ Department of Chemistry, University of Munich (LMU), Butenandtstraße 5-13, 81377 München, Germany. ${ }^{4}$ Max-Planck-Institut für Eisenforschung GmbH, Max-Planck-Straße 1, 40237 Düsseldorf, Germany. ${ }^{5}$ AMOLF, Science Park 104, 1098 XG Amsterdam, The Netherlands. ${ }^{6}$ Max-Planck-Institute for Intelligent Systems, Heisenbergstr. 3, 70569 Stuttgart, Germany. 'Institute of Physics, Faculty of Basic Sciences, EPFL, 1015 Lausanne, Switzerland. ${ }^{8}$ Nanosystems Initiative Munich (NIM), Schellingstraße 4, 80799 München, Germany. ${ }^{9}$ Center for Nanoscience, Schellingstraße 4, 80799 München, Germany.

*Corresponding author: $\underline{\text { b.lotsch@fkf.mpg.de }}$

\section{Keywords}

Catalysis, water splitting, hydrogen evolution, delafossites, dealloying, strain, phase transition, betaPalladium. 


\begin{abstract}
The rational design of hydrogen evolution reaction (HER) electrocatalysts which are competitive with platinum is an outstanding challenge to make power-to-gas technologies economically viable. Here, we introduce the delafossites $\mathrm{PdCrO}_{2}, \mathrm{PdCoO}_{2}$ and $\mathrm{PtCoO}_{2}$ as a new family of electrocatalysts for the HER in acidic media. We show that in $\mathrm{PdCoO}_{2}$ the inherently strained $\mathrm{Pd}$ metal sublattice acts as a pseudomorphic template for the growth of a strained (by $+2.3 \%$ ) Pd rich capping layer under reductive conditions. The surface modification continuously improves the electrocatalytic activity by simultaneously increasing the exchange current density $j_{0}$ from 2 to $5 \mathrm{~mA} / \mathrm{cm}^{2}$ geo and by reducing the Tafel slope down to $38 \mathrm{mV} /$ decade, leading to overpotentials $\eta_{10}<15 \mathrm{mV}$ for $10 \mathrm{~mA} / \mathrm{cm}^{2}$ geo, superior to bulk platinum. The greatly improved activity is attributed to the in-situ stabilization of a $\beta$-palladium hydride phase with drastically enhanced surface catalytic properties with respect to pure or nanostructured palladium. These findings illustrate how operando induced electrodissolution can be used as a top-down design concept for rational surface and property engineering through the strainstabilized formation of catalytically active phases.
\end{abstract}




\section{Introduction}

Global warming and the decreasing availability of fossil fuels urge today's society to transition to more sustainable energy sources. While there is enough solar and wind power to satisfy our needs in terms of total energy, 1, 2 the available power fluctuates strongly and requires intermediate and long term storage. ${ }^{3,4}$ One viable option is the storage of the intermittent electrical energy in the form of chemical fuels such as hydrogen (power-to-X). Clean hydrogen can be produced by alkaline electrolyzers, which require constant and high current densities for a stable operation. ${ }^{5}$ In acidic environments, more powerful and flexible proton exchange membrane (PEM) electrolyzers can be employed that allow for coupling with fluctuating energy sources such as wind and solar. ${ }^{6,7}$ As the most widely used electrocatalysts for the hydrogen evolution reaction (HER) is still platinum, an expensive and scarce material that is also poisoned easily, research into alternative or modified highly efficient and stable electrocatalysts under various conditions has been identified as a key goal in energy science. ${ }^{8,9,10}$

Strain effects have been discussed to be at the heart of enhanced intrinsic activities toward several catalytic reactions including the HER, as predicted and observed for example in Pd overlayers. ${ }^{11,12,13}$ More recently, the scope of this concept has been widened and the direct strain control in substrate induced strain effects or in core-shell particles have been effectively used for the HER as well as the oxygen evolution reaction (OER) and the oxygen reduction reaction (ORR). ${ }^{14,15,16,17,18,19}$

Here, we report the time evolution of the electrocatalytic activity of the $\mathrm{ABO}_{2}$ delafossites $\mathrm{PdCrO}_{2}$, $\mathrm{PdCoO}_{2}$, and $\mathrm{PtCoO}_{2}$ for the HER in acidic medium and show how inherent structural strain can be used to enhance the catalytic efficiency operando. Since their discovery and the observation of their unusually high and anisotropic conductivity in $1971,{ }^{20,21,22}$ these oxides have attracted renewed interest recently for their unusual electronic properties, ${ }^{23,24}$ anisotropic thermopower, ${ }^{25}$ and most recently, for the discovery of hydrodynamic electron flow occurring in nanostructured $\mathrm{PdCoO}_{2}{ }^{26}$ 
A common property of the metallic Pd and Pt based delafossites is an inherently expanded hexagonal metal sublattice with extended nearest neighbour distances on the A site compared to the pure metals $\left(2.830 \AA\right.$ in $\mathrm{PdCoO}_{2}$ and $2.923 \AA$ in $\mathrm{PdCrO}_{2}$ vs. $2.751 \AA$ in $f c c \mathrm{Pd}(111) ; 2.823 \AA$ in $\mathrm{PtCoO}_{2}$ vs. $2.775 \AA$ in metallic $\mathrm{Pt}(111)) .{ }^{20,21,22,24}$ This sublattice is separated by a layer of edge-sharing $\mathrm{MO}_{6}(\mathrm{M}=\mathrm{Co}, \mathrm{Cr}$ ) octahedra, see Fig. 1 a, which gives rise to anisotropic transport properties. So far, these and other delafossite oxides have been reported as highly efficient electrocatalysts for the OER in alkaline media. 27, 28, 29, 30 Furthermore, copper- and silver-based delafossites have been reported as photocathodes due to their large band gap. ${ }^{31,32,33,34}$ Apart from $\mathrm{AgRhO}_{2}$ and $\mathrm{CuCrO}_{2}$, the stability of these systems appears to be limited to basic, neutral or non-reductive conditions. ${ }^{35,36,37}$

In this work, we make use of the intrinsically strained metal sublattices and study the hydrogen evolution activity of the delafossite oxides $\mathrm{PdMO}_{2}(\mathrm{M}=\mathrm{Cr}, \mathrm{Co})$ and $\mathrm{PtCoO}_{2}$ for the first time, investigating the influence of strain on the catalytic activity. For $\mathrm{PdCoO}_{2}$, our long-term studies reveal a gradual enhancement of the already excellent water reduction activity of the bulk material over time, putting the electrocatalytic activity of operando modified $\mathrm{PdCoO}_{2}$ en par with that of the top-of-theclass HER electrocatalyst in acidic medium, platinum. We elucidate how the charge transfer properties as well as the high specific activity per surface area evolve, which we attribute to the strain-induced stabilization of in-situ formed, catalytically highly active $\beta$-palladium hydride $\left(\mathrm{PdH}_{\mathrm{x}}, \mathrm{x}^{\sim 0.62-0.67) .{ }^{38}}\right.$ Together with an apparent increase in exchange current density, these effects lead to a drastically reduced overpotential at $10 \mathrm{~mA} / \mathrm{cm}^{2}$ geo $\left(\eta_{10}\right)<15 \mathrm{mV}$ and a Tafel slope of 30-40 mV/decade, enabling stable operation at current densities of $100 \mathrm{~mA} / \mathrm{cm}^{2}$ geo with less than $100 \mathrm{mV}$ of applied (uncorrected) potential. 


\section{Results}

Electrochemical characterization. Polycrystalline powders of the delafossites $\mathrm{PdCrO}_{2}, \mathrm{PdCoO}_{2}$ and $\mathrm{PtCoO}_{2}$ were prepared according to previously reported procedures (for details, see the Method section). The crystal structure of the isostructural compounds (space group $R-3 m$ ) was confirmed by powder XRD measurements (see Fig. S1) and is schematically displayed for $\mathrm{PdCoO}_{2}$ in Fig. 1 a. ${ }^{20,39,40,41}$ The crystallite sizes were in the range from 1-3 $\mu \mathrm{m}$ for $\mathrm{PdCrO}_{2}, 10-30 \mu \mathrm{m}$ for $\mathrm{PtCoO}_{2}$ and up to $1 \mathrm{~mm}$ for $\mathrm{PdCoO}_{2}$ as evidenced by electron microscopy (see Fig. 2). These microcrystals were subsequently pressed into a carbon paste electrode (see Methods for details) to study their activity towards HER in acidic media. The analysis was performed in hydrogen saturated $1 \mathrm{M} \mathrm{H}_{2} \mathrm{SO}_{4}$ by means of cyclic voltammetry (CV), chronopotentiometry and -amperometry. The polarization curves in Fig. 1 b-d show the uncorrected cathodic currents per geometric surface area (in $\mathrm{mA} / \mathrm{cm}^{2}{ }_{\text {geo }}$ ) for the first 1000 cycles on all three materials, which all increase in efficiency in different ways. After correcting for the series resistance losses (IR-drop) extracted from impedance measurements before the respective cycles, the CVs can be fitted to the Tafel equation

$\eta=b \log \left(i / j_{0}\right)$

with $\eta$ being the overpotential with respect to the reversible hydrogen electrode (RHE), while $b$ denotes the Tafel slope (in $\mathrm{mV} /$ decade), which provides insight onto the electrocatalytic processes occurring on the surface, $i$ being the current density and $j_{0}$ the exchange current density, which is a measure of the intrinsic activity per surface area. ${ }^{42}$

The activity of all delafossites is very high, requiring an overpotential $\eta_{10}$ of far less than $100 \mathrm{mV}$ for all systems. $\mathrm{PdCrO}_{2}$ initially requires $\eta_{10}$ of approx. $50 \mathrm{mV}$ (Fig. $1 \mathrm{~b}$ and e). Subsequently, the current density decreases and stabilizes with increasing cycle number. $\mathrm{PdCoO}_{2}$ (Fig. $1 \mathrm{c}$ and e) initially also yields $\eta_{10}$ at $50 \mathrm{mV}$ vs. RHE for a similar loading and keeps improving for the first 1000 cycles down to 
12(3) $\mathrm{mV}$. In contrast, $\mathrm{PtCoO}_{2}$ initially shows a lower activity than the Pd based materials and quickly improves towards comparable activities of $\mathrm{PdCoO}_{2}$ within 30-100 cycles. Afterwards, it stabilizes at $\eta_{10}$ around 30-35 mV. Remarkably, the overpotentials of all the materials are very low after some initial cycles and especially the Co based delafossites outperform most catalysts already after 30 cycles $\left(\eta_{10}<\right.$ $40 \mathrm{mV}$ ), particularly all oxides, in acidic media. ${ }^{43,44,45,46}$

To better understand the curious evolution of the overpotential over time, we discriminate between the effects arising from the intrinsic activity and those induced operando, i.e. during the electrocatalytic process. In the first cycles, the cathodic currents are influenced by surface activation or hydrogen sorption, which is well known for Pt and Pd, respectively. ${ }^{47,48}$ This is clearly visible in the first cycle of $\mathrm{PdCrO}_{2}$ (Fig. 1 b), where the apparent high activity occurs already at the RHE potential before hydrogen saturation at the surface is achieved. In the range of $10-1000$ cycles, the catalytic mechanism on the surface evolves with increasing cycle number and the currents tend to stabilize. Interestingly, the overpotential is modified also by the catalytic process itself, which is reflected by the decreasing Tafel slopes for the Co based materials (Fig. 1f). The values between 70 and $38 \mathrm{mV} / \mathrm{dec}$ suggest a mixture between a reaction rate limited by the discharge reaction of protons at the surface $(128 \mathrm{mV} / \mathrm{dec}$, Volmer mechanism) and the Volmer-Tafel mechanism $(38 \mathrm{mV} / \mathrm{dec})$ where the recombination of adsorbed hydrogen is rate determining, which is observed after 1000 cycles on $\mathrm{PdCoO}_{2}$. Pure Pd metal, which has a high Tafel slope of $>100 \mathrm{mV} /$ decade for current densities $>1 \mathrm{~mA} / \mathrm{cm}^{2}$, is limited by the Volmer mechanism, in contrast to the delafossites presented herein, see Fig. S2. ${ }^{42,49,50}$ The observed evolution of the activity described by $\eta_{10}$ in the delafossite oxides further appears to be related to the activity of the catalysts per surface area, $j_{0}$, (Fig. $1 \mathrm{~g}$ ), which changes as a function of time and parallels the trends observed for the overpotentials shown in Fig. 1 e. While $j_{0}$ tends to slightly decrease for $\mathrm{PdCrO}_{2}$, it increases for both Co containing materials, indicating an effective increase in the intrinsic activity or catalytically active area, or both. The values for $j_{0}$ evolving with time are all in the range of 
$1 \mathrm{~mA} / \mathrm{cm}^{2}$ and thus amongst the highest reported to date. For $\mathrm{PdCoO}_{2}, j_{0}$ of up to $5(1.2) \mathrm{mA} / \mathrm{cm}^{2}$ is even superior to those observed for both bulk Pt and $\mathrm{Pd}\left(2.3\right.$ and $0.9 \mathrm{~mA} / \mathrm{cm}^{2}$, respectively), as shown in Fig. $\mathrm{S} 2 .{ }^{42,43,51}$

In order to obtain an estimate of the real surface area, impedance measurements were performed to extract the double layer capacitance $C_{D L}$, which is proportional to the electrochemical surface area (ECSA), and the pseudocapacitive contribution $\mathrm{C}_{\mathrm{P}}$ (see discussion in the SI, section 3 and Fig. S3). Since due to their modified surface properties no references for the operando modified delafossites are available, the same capacitive response factors as for bulk Pd or Pt were assumed (see SI section 3 for details). We then obtain an ECSA normalized $\mathrm{j}_{0, \mathrm{ECSA}}$ of $0.14 \mathrm{~mA} / \mathrm{cm}^{2}$ for $\mathrm{PdCrO}_{2}$ and $0.20 \mathrm{~mA} / \mathrm{cm}^{2}$ for $(\mathrm{Pd}, \mathrm{Pt}) \mathrm{CoO}_{2}$ after 300 cycles (see Fig. S3 for details). These values are close to dealloyed Pd nanoparticles without normalization for real surface area $\left(0.18 \mathrm{~mA} / \mathrm{cm}^{2}\right)^{52}$ and reported surface normalized values of $0.14 \mathrm{~mA} / \mathrm{cm}^{2}$ for tensile strained $\mathrm{Pd}$ grown on Au or rough Pd surfaces $(0.22$ $\left.\mathrm{mA} / \mathrm{cm}^{2}\right) .{ }^{53,54}$

a
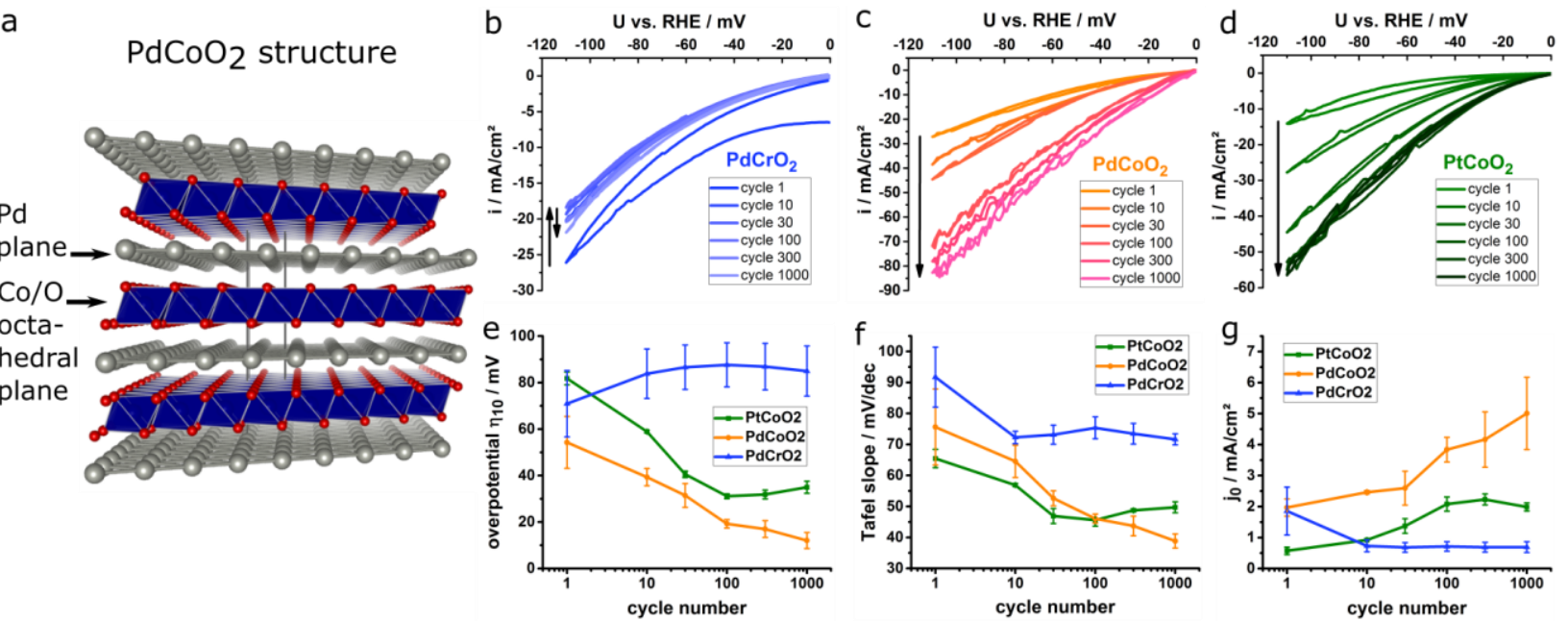

Figure 1: Crystal structure of the delafossites and the evolution of the electrochemical activity for HER. a: The layered delafossite structure using $\mathrm{PdCoO}_{2}$ as a representative example, view along [100] (Pd: grey; oxygen: red, Co: blue octahedra). bd: Uncorrected cathodic currents for $\mathrm{PdCrO}_{2}$ (b), $\mathrm{PdCoO}_{2}$ (c) and $\mathrm{PtCoO}_{2}$ (d) in hydrogen saturated $1 \mathrm{M} \mathrm{H}_{2} \mathrm{SO}_{4}$ during cathodic cycling (scan rate: $2 \mathrm{mV} / \mathrm{s}$ for displayed measurements, $25 \mathrm{mV} / \mathrm{s}$ for aging in-between the displaced cycles). d-f: The resulting 
Tafel parameters (e: overpotential $\eta_{10}, \mathrm{f}$ : Tafel slope, and g: exchange current density for geometric surface area) after IRcorrection and fitting in the region $>1 \mathrm{~mA} / \mathrm{cm}^{2}$.

To provide insights into the origin of the currents observed in the electrocatalytic experiments, the evolution of hydrogen was investigated close to the surface by scanning electrochemical microscopy (SECM) measurements on $\mathrm{PdCoO}_{2}$ single crystals operando (see Fig. S4 a \& b for illustration). Although no site dependence could be mapped due to a tip-limited resolution $>10 \mu \mathrm{m}$, the probing electrode feedback clearly shows the presence of hydrogen near the sample surface ( $10 \mu \mathrm{m})$ when the sample is held at different cathodic sample potentials under weakly acidic conditions. Cyclic voltammograms reflecting the reductive SECM tip HER current for different sample potentials show a decrease when the $\mathrm{PdCoO}_{2}$ sample HER current becomes competitive, and an increase in the hydrogen oxidation current at oxidative tip potentials (Fig. S4 c). Temporally, an increase in hydrogen production at the sample could be evidenced by increasing proton reduction currents at the tip (Fig. S4 d). This highlights the fact that pristine samples are HER active and improve over time, even without cycling. Furthermore, a comparison of the charge passed through a pre-aged $\mathrm{PdCoO}_{2}$ single crystal ( $>1000$ cycles), referenced to a Pt wire, yielded stable Faradaic efficiencies towards hydrogen evolution of $>90 \%$. In contrast, a pure Pd wire had a Faradaic efficiency of only 6\%, probably due to large amounts of $\mathrm{H}_{2}$ being incorporated into the Pd lattice, even after long coulometric measurements (see Fig. S5). ${ }^{50,51}$

Surface modification analysis. Next, to understand the origin of the observed increase in catalytic activity over time, the modifications on the surface of the delafossites were tracked by analysis of the electrolyte composition by means of inductively coupled plasma optical emission spectrometry (ICPOES) after the electrocatalytic experiments (see SI Table ST1). Almost no detectable amounts of $\mathrm{Cr}$ in the electrolyte suggest a high stability of this element in the $\mathrm{PdCrO}_{2}$ surface $(<0.08$ at\% of the $\mathrm{Cr}$ amount present in the electrode), while $\mathrm{PtCoO}_{2}$ showed small amounts of $\mathrm{Co}$ in the electrolyte, corresponding to 0.24(3) at\% of the electrode element. No dissolved Pt was evidenced in all cases. In contrast, $\mathrm{PdCoO}_{2}$ 
shows increased amounts of Co in the electrolyte, indicating a much faster dissolution of Co, which progresses at a rate two orders of magnitude faster than $\mathrm{Cr}$ dissolution. ${ }^{55}$ After 1000 cycles, 9.35(90) at\% of the Co has leached out from $\mathrm{PdCoO}_{2}$.

Consequently, we expect the formation of a $\mathrm{Pd}$ rich surface layer on $\mathrm{PdCoO}_{2}$. Indeed, direct evidence of the surface transformation is obtained by optical microscopy and scanning electron microscopy (SEM) on the surfaces of the delafossites after catalysis. While $\mathrm{PdCrO}_{2}$ does not show any obvious signs of corrosion (Fig. 2 a-b), the surface of $\mathrm{PtCoO}_{2}$ is slightly modified, which is elucidated by a slight roughening and accumulation of heavier elements as shown by the back scattered electron (BSE) detector image (Fig. $2 \mathrm{c}$-d vs e-f). In contrast, the surface of $\mathrm{PdCoO}_{2}$ is strongly modified, as shown in Fig. $2 \mathrm{~g}-\mathrm{I}$ and mirrored by the significant Co dissolution and a stronger change in catalytic properties. Inspection under an optical microscope (Fig. 2 g-h) reveals a large area surface modification and roughening. A more detailed SEM analysis of the surface (Fig. 2 i vs j) shows the formation of a continuous capping layer with a higher concentration of heavy elements than the pristine, underlying material. Energy dispersive X-ray spectroscopy (EDS) maps on the modified surface further underline the enrichment of Pd in this topmost capping layer. The formation of cracks and nanoparticles within the capping layer (Fig. $2 \mathrm{j}-1$ ) suggests a release of strain still present in the remaining capping structure after Co and $\mathrm{O}$ are dissolved near the surface (vide infra). These cracks can also be related to potential dependent hydrogen loading and unloading into the catalysts during electrocatalytic cycling, which results in dynamically modified lattice parameters. ${ }^{56}$ Also, small amounts of Pd were found in the electrolyte after catalysis, which can be explained by partial detachment of the strained capping layer (Fig. S6 a-c). ${ }^{56}$ 


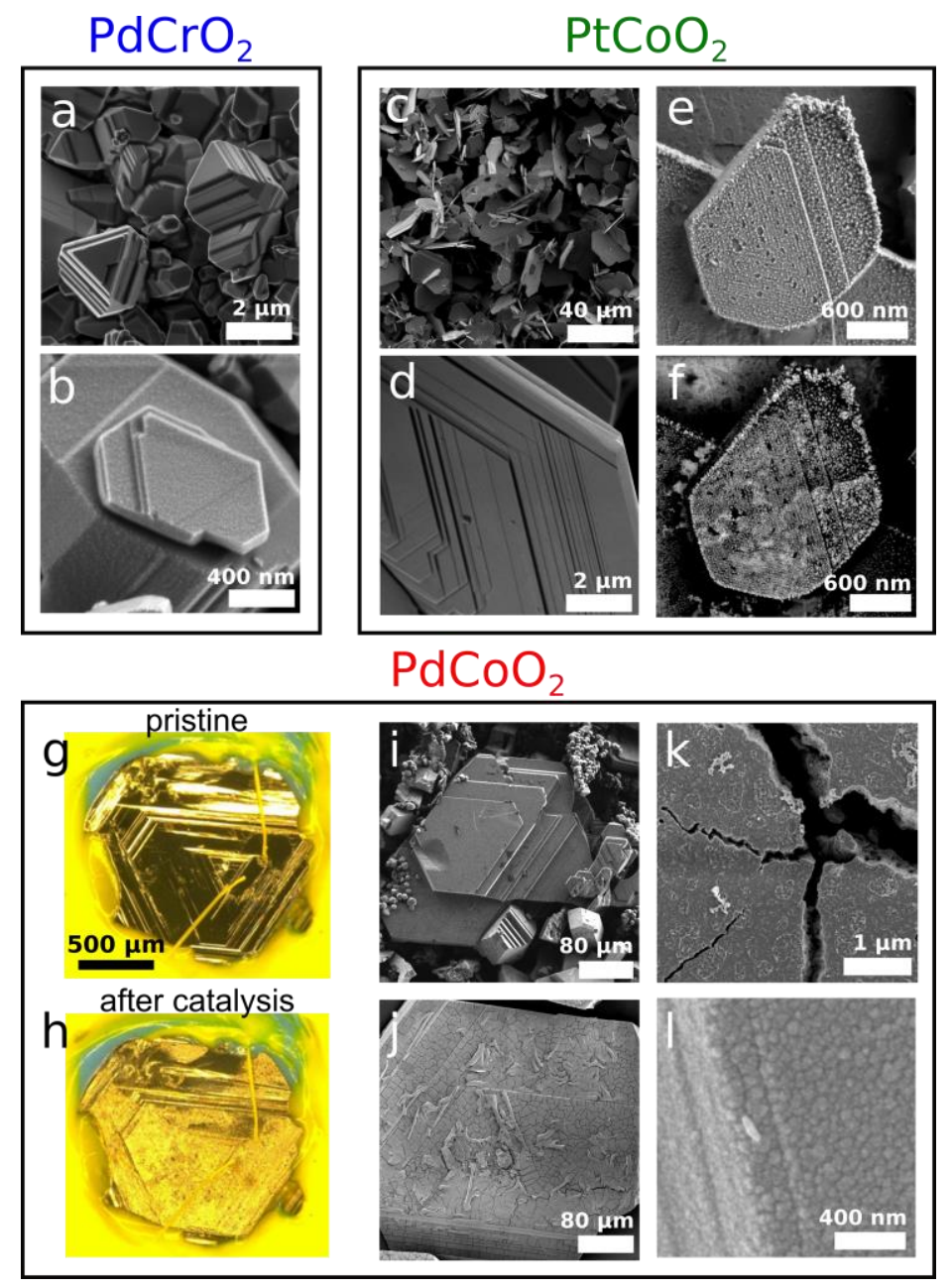

Figure 2: Surface modifications on the delafossites before and after electrocatalytic $\mathrm{HER}$ in $1 \mathrm{M} \mathrm{H}_{2} \mathrm{SO}_{4}$. a: $\mathrm{PdCrO}_{2} \mathrm{crystals}$ assynthesized. b: Enlarged image after cycling. c-d: As-synthesized $\mathrm{PtCoO}_{2}$ crystals. e-f: PtCoO2 after cycling. The BSE detector image (f) highlights the heavy element nature of the capping layer, containing mostly Pt. g-h: Optical microscope images of a large $\mathrm{PdCoO}_{2}$ crystal before and after catalysis, embedded in yellow sealing. i: SEM image of a large $\mathrm{PdCoO}_{2} \mathrm{crystal}_{\text {embedded }}$ in a carbon paste electrode prior to cycling. j: $\mathrm{PdCoO}_{2}$ surface modification after cycling showing the Pd rich capping layer. k: Zoom into the cracked surface structure. I: Magnification of the rough nanostructure on the surface.

To further investigate the surface modification, X-ray photoelectron spectroscopy (XPS) was used to measure the valence state of the respective components of all three materials before and after catalysis, as shown in Fig 3. A detailed discussion including survey spectra (Fig. S7) and peak lists (Tables ST2-5) can be found in the SI section 8. In brief, the main elemental lines of all pristine delafossites correspond well to the literature values. In the oxygen spectra (Fig. $3 \mathrm{c}, \mathrm{f}$ and $\mathrm{i}$ ), an $\mathrm{O} 1 \mathrm{~s}$ signal at $531-532 \mathrm{eV}$ is 
attributed to surface adsorbed oxygen. After catalysis, the amplitude of the delafossite M-O interaction peak at $529 \mathrm{eV}$ is slightly lowered relative to the surface $\mathrm{O}$ 1s peak in $\mathrm{PdCrO}_{2}$ (Fig. 3c), suggesting somewhat decreasing binding of $\mathrm{Pd}$ and $\mathrm{Cr}$ to $\mathrm{O}$ at the surface. For $\mathrm{PdCoO}_{2}$ (Fig. $3 \mathrm{~d}$-f) and $\mathrm{PtCoO}_{2}$ (Fig. 3 $\mathrm{g}-\mathrm{i})$, the Co as well as the M-O 1s signals completely disappear after catalysis, with slightly slower kinetics for $\mathrm{PtCoO}_{2}$ (see Fig. S8), underlining the formation of a $\mathrm{Pd}(0)$ or $\mathrm{Pt}(0)$ layer on the surface during catalysis, in agreement with the ICP measurements on Co dissolution. Summarizing these results, we can conclude that $\mathrm{PdCrO}_{2}$ remains largely unchanged over the cycling experiments, while $\mathrm{PtCoO}_{2}$ and $\mathrm{PdCoO}_{2}$ gradually lose $\mathrm{Co}$ and are eventually reduced, forming a metallic capping layer of the respective noble metal. 

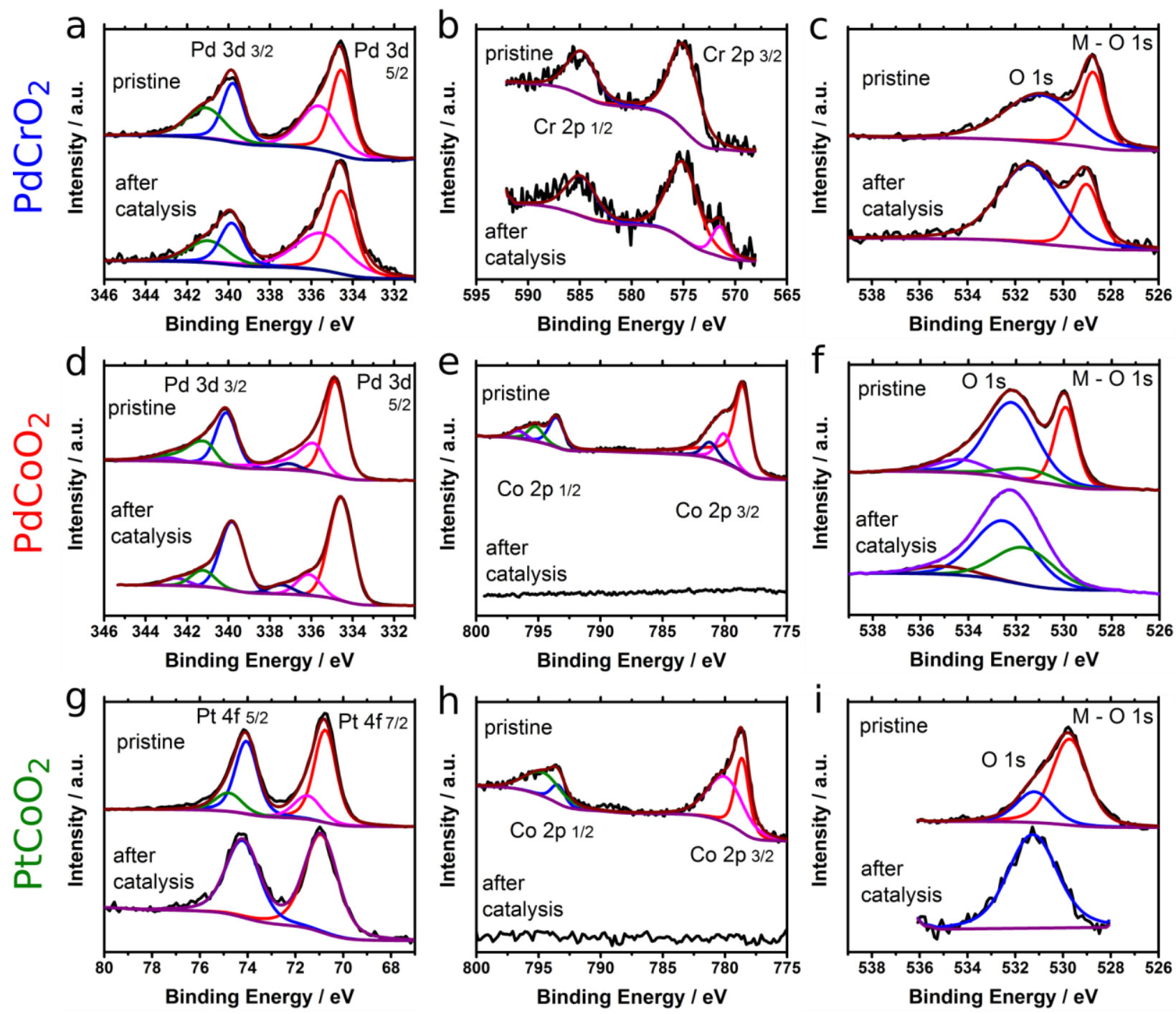

Figure 3: XPS spectra of $\mathrm{PdCrO}_{2}(\mathrm{a}-\mathrm{c}), \mathrm{PdCoO}_{2}(\mathrm{~d}-\mathrm{f})$ and $\mathrm{PtCoO}_{2}(\mathrm{~g}-\mathrm{i})$ before (top trace) and after (bottom trace) catalytic cycling in $1 \mathrm{M} \mathrm{H}_{2} \mathrm{SO}_{4}$. While the surface of $\mathrm{PdCrO}_{2}$ remains almost unchanged, the surface of $\mathrm{PdCoO}_{2}$ is transformed into $\mathrm{Pd}$ with traces of oxygen adsorbed to the surface. For $\mathrm{PtCoO}_{2}$, the behavior is similar and a Pt rich surface is obtained after longer aging. A peak list including all signals and their areas as well as the survey and additional spectra are shown in the SI section 8 (Fig. S7-8, Table ST 2-5).

Capping structure and strain analysis. Having identified the nature of the surface corrosion process as the reductive formation of a $\mathrm{Pd}$ rich capping layer on $\mathrm{PdCOO}_{2}$, we turn to the question as to why this process leads to the observed exceptionally high activity towards HER. To this end, we first used scanning transmission electron microscopy (STEM) to characterize the structure and elemental 
composition of the pre- and post-catalysis samples at high spatial resolution. STEM lamellae were prepared from a $\mathrm{PdCoO}_{2}$ single crystal after short $(5 \mathrm{~min}$ ) and long (hours) chronoamperometric aging at $-100 \mathrm{mV}$ vs. RHE, which both lead to the formation of a capping layer with increasing thickness (see Fig. 2 c-e and S6). A cross section lamella of a 5 min aged single crystal contains both the capping layer and the underlying material, as shown in Fig. 4 (see SI section 6 for experimental details and Fig. S 6 for a lamella prepared after hours of aging). The Pd capping layer is ca. $30 \mathrm{~nm}$ thick and consists of Pd nanocrystals of 2-10 nm size (Fig. 4 a-d). Elemental analysis of the capping layer by EDS spectrum imaging ${ }^{57}$ shows that the cap consists primarily of Pd (Fig. 4 e), while the Co concentration is below the detection limit $(<2 \%)$. $\mathrm{O}$ is only observed at the surface of the $\mathrm{Pd}$ capping layer. The concentration profiles are summarized in the right display element of Fig. $4 \mathrm{f}$. As shown in Fig. 4 a, the Pd fcc capping layer grows directly on the $\mathrm{PdCoO}_{2}$ surface, in direct contact to the electrolyte. There is a sharp transition between the $\mathrm{PdCoO}_{2}$ crystal and the Pd cap (Fig. 4 b). The atomic resolution STEM-high angle annular dark field (HAADF) image of $\mathrm{PdCoO}_{2}$ (Fig. 4 c) is overlaid by the crystal structure along the [1120] zone axis. We observe a preferred orientation of the cap on the $\mathrm{PdCoO}_{2}$ substrate, suggesting quasiepitaxial growth of the $\mathrm{Pd}(111)$ in the c-direction of the bulk $\mathrm{PdCoO}_{2}$ (normal to the hexagonal Pd sublattice). Within the cap structure, twin variants of Pd nanocrystals are observed (Fig. $4 \mathrm{~d}$ and S9). Fast Fourier Transformations (FFT) of the respective cap ( $\mathrm{Pd} f c c)$ and bulk $\left(\mathrm{PdCoO}_{2}\right)$ regions reveal only a few degrees tilt between $\mathrm{Pd}(111)$ and $\mathrm{PdCoO}_{2}$ (0003), as shown in Fig. S9 (a and b). This lends further evidence that the capping layer grows via leaching of Co and O from the bulk material, while the hexagonal $\mathrm{Pd}$ lattice in $\mathrm{PdCoO}_{2}$ evolves into an $f c c$ Pd lattice with minimal structural reorganization, as illustrated in Fig. 4 g. To derive the lattice parameter of the Pd nanocrystals, STEM micrographs were calibrated using the lattice parameters of $\mathrm{PdCoO}_{2}$ from XRD measurements. Compared to the sharp reflections of $\mathrm{PdCOO}_{2}, \mathrm{Pd}$ reflections are broader along the radial and azimuthal axes, corresponding to a broader range of strain and rotation among Pd nanocrystals. Analysis of all $\mathrm{Pd}\{111\}$ reflections evidence 
a homogeneous lattice parameter of $3.98 \AA$ on average, which proves that the $\mathrm{Pd} f c c$ capping layer shows isotropic tensile strain, even for both twin variants (Fig. S9 c). Further comparison shows a slightly smaller lattice parameter at the top $5 \mathrm{~nm}$ of the cap than the bottom part (Fig. S9 e-g), suggesting partial strain relaxation towards the surface, especially for the $\mathrm{Pd}(111)$ planes that are parallel to the $\mathrm{PdCoO}_{2}$ surface (Fig. S8, d-g). This is expected for quasi-epitaxial growth and partial strain release due to cracks. Nevertheless, the lattice parameters close to the surface are still larger than those of bulk Pd crystals (3.89 ̊̊) ${ }^{58}$ or the ones reported for Pd nanoparticles with similar size (approx. $10 \mathrm{~nm}$ ) that show no lattice dilatation (see also Fig. S10). ${ }^{59}$ Only below $3 \mathrm{~nm}$, a comparable lattice parameter has been reported for pure Pd nanoparticles deposited on carbon. ${ }^{60}$ A lattice constant of $3.98 \AA$ in the capping layer corresponds to Pd nearest neighbour distances of $2.815 \AA$ in the Pd fcc structure, which is very close to $2.83 \AA$ in the Pd sublattice of $\mathrm{PdCoO}_{2}$ and significantly differs from unstrained Pd $(2.75 \AA)$. This further underlines the relevance of lattice strain in the substrate material, $\mathrm{PdCoO}_{2}$, which is transduced to the capping layer.

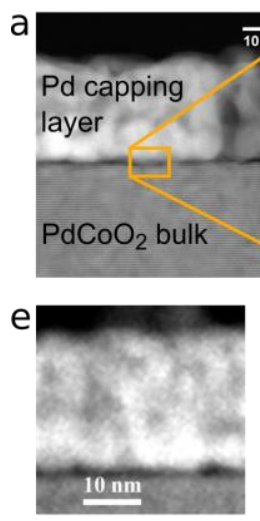

STEM-HAADF
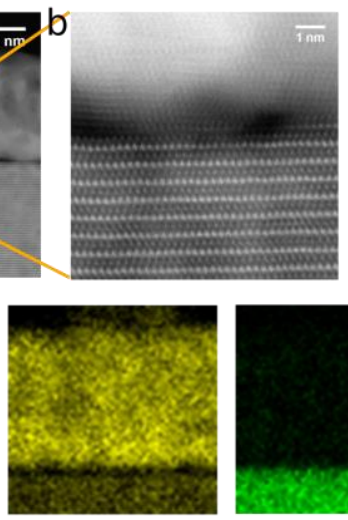

Pd-L

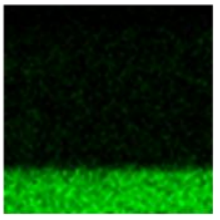

Co-K
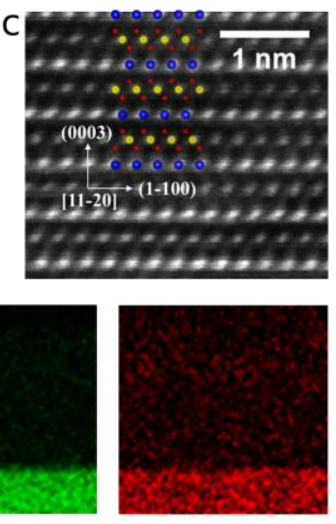

O-K
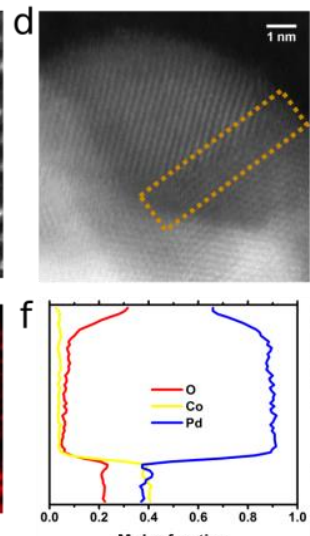

Molar fraction

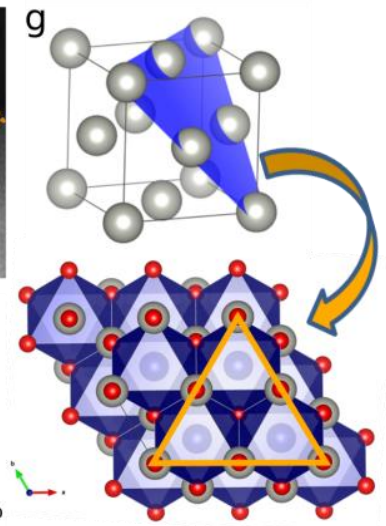

Figure 4: $\mathrm{STEM}$ analysis of the $\mathrm{Pd}$ capping layer on $\mathrm{PdCoO}_{2}$. a: $\mathrm{STEM}-\mathrm{HAADF}$ micrograph of $\mathrm{PdCoO}_{2}$ after $5 \mathrm{~min}$ aging at $\eta=100$ $\mathrm{mV}$ in $1 \mathrm{M} \mathrm{H}_{2} \mathrm{SO}_{4}$, showing the bulk material and the Pd rich capping layer. b: $\mathrm{PdCoO}_{2}$ atomic structure (Pd: blue, Co: yellow, O: red). c: Pd fcc nanocrystals in the overlayer containing twinned grain boundaries (marked in orange). d: STEM-HAADF image and respective STEM-EDS elemental maps based on the Pd-L line (blue), the Co-K line (yellow) and O-K line (red) and a line profile of 
their molar fractions (right element). e: Illustration of the Pd fcc structure with the (111) plane corresponding to the hexagonal Pd sublayers in $\mathrm{PdCoO}_{2}$, which act as a growth template for $\mathrm{Pd} f c c$.

The observations made on $\mathrm{PdCoO}_{2}$ in Fig. 4 agree with ICP measurements of dissolved Co in the electrolyte and the XPS data (Fig. 3) but further suggest that the continuous dissolution of Co and 0 creates a significantly strained Pd-rich capping layer, where the strain is transmitted by the pseudomorphic growth of $\mathrm{Pd}(111)$ on the hexagonal $\mathrm{Pd}$ sublattice in $\mathrm{PdCoO}_{2}$.

Pd nanoparticle reference measurements. Since the particle size and density of surface states of Pd nanocrystals in the capping layer $(2-10 \mathrm{~nm})$ differs from that of bulk Pd, which can affect the catalytic activity, $^{59,61}$ we studied the electrochemical activity of Pd nanoparticles of comparable size (1 - $10 \mathrm{~nm}$, Sciventions) to gauge the effect of the particle morphology and strain on the catalytic activity (see Fig. S10-11). Rietveld refined XRD measurements of the dried reference nanoparticles revealed an average lattice constant of $3.90(2) \AA$ (see Fig. S10), which fits reported values for bulk Pd (3.89 $\AA$ ) and Pd nanostructures of $8-10 \mathrm{~nm}$ size. ${ }^{59}$ The lattice constant of the nanoparticles is thus $2 \%$ smaller than for the nanocrystals at the bottom of the Pd capping layer (3.98 A). Since a comparable surface coverage and active surface area is difficult to achieve, the catalytic properties of both bulk Pd and the nanoparticles are best characterized by their Tafel slope, which is only sensitive to the underlying catalytic process and independent of the actual effective surface area. ${ }^{42}$ Both the nanoparticles and the bulk Pd show a similar Tafel slope of $105 \mathrm{mV} / \mathrm{dec}$ (Fig. S11 and S2), which is consistent with the Pd literature value of $103 \mathrm{mV} / \mathrm{dec}^{49}$ and relates the surface catalytic process to a rate limiting Volmer (discharge) reaction. This value is much larger than the values found for surface modified $\mathrm{PdCoO}_{2}$ in $\mathrm{Fig}$. 1 and S2 (38 to $30 \mathrm{mV} / \mathrm{dec}$ ), which suggests a rate limiting Volmer-Heyrovsky or -Tafel mechanism (38 and $30 \mathrm{mV} / \mathrm{dec}$, respectively). ${ }^{42,62}$ The intrinsic catalytic mechanism of the strained Pd capping layer is thus different from pure Pd, regardless of particle size or morphology. 
Discussion. To rationalize the observed tensile strain-induced activity increase in $\mathrm{PdCoO}_{2}$, we first consider the effect of strain as expected from classical d-band theory, which predicts a correlation between the position of the metal $d$-band center with the HER activity. ${ }^{53}$ The relative energies of the metal $\mathrm{d}$-band and the antibonding hydrogen $\sigma^{*}$-orbital directly determine their overlap and, hence, bonding strength of the $\mathrm{H}$-adsorbate to the metal surface, $\Delta \mathrm{G}_{\mathrm{H}}{ }^{63}$ Following Sabatier's principle, the catalyst - substrate interaction should be neither too strong nor too weak such that both adsorption of the substrate and desorption of the product is facile. Hence, $\Delta G_{H}$ is used as the most relevant descriptor of the intrinsic activity for HER, given by a turnover frequency or $j_{0}$, resulting in a volcano plot, with optimal catalyst - substrate interactions at the top of the volcano. ${ }^{42,64}$ Tensile lattice strain in Pd tends to flatten out the d-bands while upshifting the band center towards the Fermi level, leading to stronger overlap with the hydrogen $\sigma^{*}$-orbital and, thus, stronger metal $-\mathrm{H}$ bonds. ${ }^{12,}{ }^{65}$ In fact, growth of a pseudomorphic Pd layer on a single crystalline substrate with a larger lattice constant has been shown to increase $\Delta G_{H}$ and thus move tensile strained Pd away from the top of the volcano while decreasing the exchange current density. ${ }^{13,53}$ Interestingly, we observe the opposite trend, enhancing both $j_{0}$ and reducing the Tafel slope. The observed change in Tafel slope by a factor of almost 3 with respect to bulk Pd suggests a more fundamental change in the materials surface properties to be at play. Indeed, tensile strain positively affects the hydrogen adsorption capacity of $\mathrm{Pd}$ and has been described to modify the potential of hydrogen adsorption in Pd. ${ }^{53,66,67,68}$ On the other hand, the different lattice parameters between hydrogen poor $\alpha-P d$ and the hydrogen rich $\beta$-phase (sharp transition and discontinuous lattice parameter transition from 3.89 to $4.02 \AA$ ) kinetically hinder a phase transformation. ${ }^{69,70,71}$ Since our expanded Pd fcc lattice intrinsically shows stable lattice parameters (as probed by TEM after prolonged ex situ sample storage) close to those of $\beta-\mathrm{PdH}_{x}\left(3.98 \AA\right.$ and $4.02 \AA$, respectively), ${ }^{71,72}$ incorporation of 
hydrogen is expected to be more facile. ${ }^{66}$ This conjecture rationalizes the corresponding findings on the Tafel slopes, which indeed show $\beta-\mathrm{PdH}_{\mathrm{x}}$-like behavior (30-38 mV/dec Tafel slope). ${ }^{51,62}$ To investigate the possible operando formation of a hydride phase, faster cyclic voltammetry measurements $(25 \mathrm{mV} / \mathrm{sec}$ and $100 \mathrm{mV} / \mathrm{sec}$ ) were recorded after 1000 cycles (Fig. S 12). With increasing scan rate, a stronger capacitive contribution is observed. It is most prominent for $\mathrm{PdCoO}_{2}$, as evidenced by the separation of the forward and backward scans around the open circuit potential (OCP), and the OCP shifts to more cathodic potentials for this material (Fig. S12 b, 100mV/s scans). This indicates, in accordance with the impedance data presented in Fig. S3, that the capping layer is active for hydrogen adsorption - a wellknown phenomenon in $\mathrm{Pd}$ metal that forms interstitial hydrides $\left(\mathrm{PdH}_{\mathrm{x}}\right) .{ }^{48,50,51}$ Since the sorption effects are very fast $(\sim 1 \mathrm{~s})$, the transition to $\beta-\mathrm{PdH}_{\mathrm{x}}$ can occur almost immediately once a strained $\mathrm{Pd}$ capping layer has been formed under reductive conditions.

In summary, we have investigated the delafossite oxides $\mathrm{PdCrO}_{2}, \mathrm{PdCoO}_{2}$, and $\mathrm{PtCoO}_{2}$ for $\mathrm{HER}$ activity in acidic media for the first time. In their pristine form these materials outperform most reported electrocatalysts with respect to their overpotentials required for $10 \mathrm{~mA} / \mathrm{cm}^{2}{ }_{\text {geo }}$ (71(15) $\mathrm{mV}$ for $\mathrm{PdCrO}_{2}$, 54(12) $\mathrm{mV}$ for $\mathrm{PdCoO}_{2}, 82(3) \mathrm{mV}$ for $\mathrm{PtCoO}_{2}$ in the first cycles). The very high activity for HER originates from a superior intrinsic surface activity, translating into exchange current densities on the order of $\mathrm{mA} / \mathrm{cm}^{2}$ (initially $1.9(8) \mathrm{mA} / \mathrm{cm}^{2}$ for $\mathrm{PdCrO}_{2}, 2.0(3) \mathrm{mA} / \mathrm{cm}^{2}$ for $\mathrm{PdCoO}_{2}, 0.57(12) \mathrm{mA} / \mathrm{cm}^{2}$ for $\mathrm{PtCoO}_{2}$ ), which are orders of magnitude higher than most HER catalysts besides Pt. This property is especially beneficial for HER at small overpotentials, where the effect of activity increase by the Tafel slope does not yet come into play. While $\mathrm{PdCrO}_{2}$ is relatively stable under acid HER conditions, the surfaces of $\mathrm{PtCoO}_{2}$ and especially $\mathrm{PdCoO}_{2}$ corrode over time via the reductive dissolution of $\mathrm{Co}$ and $\mathrm{O}$ at the surface near region. The resulting $\mathrm{Pd}$ or Pt enriched capping layers show significantly enhanced activities compared to the bulk materials: $j_{0}$ increases by a factor of 2.5 and 3.5 for $\mathrm{PdCoO}_{2}$ and $\mathrm{PtCoO}_{2}$, while the Tafel slopes decrease from 76(13) to $38(3) \mathrm{mV} / \mathrm{dec}$ and from $65(3)$ to $50(2) \mathrm{mV} / \mathrm{dec}$, respectively. 
In the case of $\mathrm{PdCOO}_{2}$, the tensile strain, which is present in the noble metal sublattice of all these delafossites, is translated to the Pd $f c c$ capping layer, which grows directly on the underlying bulk material. This strain increases the hydrogen adsorption energy as predicted by d-band theory and lowers the energetic barrier for the formation of a $\beta-\mathrm{PdH}_{x}$ phase, which otherwise requires a significant lattice expansion (+3.3\%). This phase transformation entails a change in HER mechanism, shifting the surface catalytic process away from the rate limiting Volmer-reaction found in Pd towards the more efficient Volmer-Tafel or Volmer-Heyrovsky mechanism. The resulting material has an overpotential of 12(3) $\mathrm{mV}$ for $10 \mathrm{~mA} / \mathrm{cm}^{2}$ geo only, hence outperforming even bulk Pt.

The herein presented strain engineering points towards a more general design principle for the rational activity enhancement in electrocatalysts with inherently strained metal sublattices. It further suggests that phase transformations, stabilized by strain, may be key players in determining both metalsubstrate interactions and the intrinsic catalytic activity, and as such, strain-induced phase changes may complement classical d-band theory as a model to predict catalytic performance.

\section{Acknowledgements}

We gratefully acknowledge Peter Schützendübe and Michaela Wieland for XPS measurements, Eleonora Frau and Pranit lyengar for the introduction to and assistance with SECM measurements. Y. Eren Suyolcu, Aliaksandr Bandarenka and especially Rotraut Merkle are acknowledged for fruitful discussions. E.A.L. acknowledges the support from the SNF Ambizione Energy program and the research program of FOM, which is financially supported by The Netherlands Organization for Scientific Research (NWO). S.Z. and C.S. acknowledge financial support from the German Research Foundation DFG under the priority programme SPP 1613 [DFG SCHE 634/12-2]. F.P, E.A.L, B.V.L and A.F.M thank the MPS-EPFL center for financial and logistic support. 


\section{Data availability}

The data supporting the plots within this paper and other findings of this study are available from the corresponding author upon reasonable request.

\section{Author contributions}

F.P., D.W., F.H. and B.V.L conceived the project and the contributing measurements. The materials were synthesized by D.W., L.D. and R.E. All sample preparation and electrochemical measurements were done by F.P. The SECM data was analyzed and discussed by E.A.L and F.P. G.R. and F.P. analyzed the XPS data. S.Z. performed the STEM experiments including the data analysis and presentation. F.P. created all other graphs. F.P. and B.V.L wrote the manuscript. All authors including C.S. and A.F.M. contributed to the discussion of the measurements, the data interpretation and the manuscript discussion.

\section{Conflict of interest}

The authors declare no conflict of interest. 


\section{Literature}

1. Lewis NS, Nocera DG. Powering the planet: Chemical challenges in solar energy utilization. Proceedings of the National Academy of Sciences 2006, 103(43): 1572915735.

2. Lewis NS. Toward Cost-Effective Solar Energy Use. Science 2007, 315(5813): 798-801.

3. Podjaski F, Kröger J, Lotsch BV. Toward an Aqueous Solar Battery: Direct Electrochemical Storage of Solar Energy in Carbon Nitrides. Advanced Materials 2018, 30(9): 1705477.

4. Yu MZ, McCulloch WD, Huang ZJ, Trang BB, Lu J, Amine K, et al. Solar-powered electrochemical energy storage: an alternative to solar fuels. Journal of Materials Chemistry A 2016, 4(8): 2766-2782.

5. Gülzow E. Alkaline fuel cells: a critical view. Journal of Power Sources 1996, 61(1): 99104.

6. Tobias R, Nhan NH, Detre T, Robert S, Peter S. Electrocatalytic Oxygen Evolution Reaction in Acidic Environments - Reaction Mechanisms and Catalysts. Advanced Energy Materials 2017, 7(1): 1601275.

7. Carmo M, Fritz DL, Mergel J, Stolten D. A comprehensive review on PEM water electrolysis. International Journal of Hydrogen Energy 2013, 38(12): 4901-4934.

8. Vesborg PCK, Jaramillo TF. Addressing the terawatt challenge: scalability in the supply of chemical elements for renewable energy. RSC Advances 2012, 2(21): 7933-7947.

9. Electrocatalysis in Fuel Cells. Springer: London, 2013.

10. Papageorgopoulos DC, Keijzer M, Veldhuis JBJ, de Bruijn FA. CO tolerance of Pd-rich platinum palladium carbon-supported electrocatalysts - Proton exchange membrane fuel cell applications. Journal of the Electrochemical Society 2002, 149(11): A1400A1404. 
11. Mavrikakis M, Hammer B, Nørskov JK. Effect of Strain on the Reactivity of Metal Surfaces. Physical Review Letters 1998, 81(13): 2819-2822.

12. Kibler LA, El-Aziz AM, Hoyer R, Kolb DM. Tuning Reaction Rates by Lateral Strain in a Palladium Monolayer. Angewandte Chemie International Edition 2005, 44(14): 20802084.

13. Greeley J, Jaramillo TF, Bonde J, Chorkendorff I, Nørskov JK. Computational highthroughput screening of electrocatalytic materials for hydrogen evolution. Nature Materials 2006, 5: 909.

14. Strasser P, Koh S, Anniyev T, Greeley J, More K, Yu C, et al. Lattice-strain control of the activity in dealloyed core-shell fuel cell catalysts. Nature Chemistry 2010, 2: 454.

15. Du M, Cui L, Cao Y, Bard AJ. Mechanoelectrochemical Catalysis of the Effect of Elastic Strain on a Platinum Nanofilm for the ORR Exerted by a Shape Memory Alloy Substrate. Journal of the American Chemical Society 2015, 137(23): 7397-7403.

16. Putungan DB, Lin S-H, Kuo J-L. A first-principles examination of conducting monolayer $1 \mathrm{~T}^{\prime}-\mathrm{MX} 2(\mathrm{M}=\mathrm{Mo}, \mathrm{W} ; \mathrm{X}=\mathrm{S}, \mathrm{Se}, \mathrm{Te})$ : promising catalysts for hydrogen evolution reaction and its enhancement by strain. Physical Chemistry Chemical Physics 2015, 17(33): 21702-21708.

17. Escudero-Escribano $M$, Malacrida $\mathrm{P}$, Hansen $\mathrm{MH}$, Vej-Hansen UG, Velázquez-Palenzuela A, Tripkovic V, et al. Tuning the activity of Pt alloy electrocatalysts by means of the lanthanide contraction. Science 2016, 352(6281): 73-76.

18. Wang H, Xu S, Tsai C, Li Y, Liu C, Zhao J, et al. Direct and continuous strain control of catalysts with tunable battery electrode materials. Science 2016, 354(6315): 1031-1036.

19. Luo M, Guo S. Strain-controlled electrocatalysis on multimetallic nanomaterials. Nature Reviews Materials 2017, 2: 17059.

20. Shannon RD, Rogers DB, Prewitt CT. Chemistry of Noble Metal Oxides. I. Syntheses and Properties of ABO2 Delafossite Compounds. Inorg Chem 1971, 10(4): 713. 
21. Prewitt CT, Shannon RD, Rogers DB. Chemistry of Noble Metal Oxides. II. Crystal Structures of PtCoO2, PdCoO2, CuFeO2, and AgFeO2. Inorg Chem 1971, 10(4): 719-723.

22. Rogers DB, Shannon RD, Prewitt CT, Gillson JL. Chemistry of Noble Metal Oxides. III. Electrical Transport Properties and Crystal Chemistry of $\mathrm{ABO} 2$ Compounds with Delafossite Structure. Inorg Chem 1971, 10(4): 723-\&.

23. Mackenzie AP. The properties of ultrapure delafossite metals. Reports on Progress in Physics 2017, 80(3): 032501.

24. Daou R, Frésard R, Eyert V, Hébert S, Maignan A. Unconventional aspects of electronic transport in delafossite oxides. Science and Technology of Advanced Materials 2017, 18(1): 919-938.

25. Ong KP, Singh DJ, Wu P. Unusual Transport and Strongly Anisotropic Thermopower in PtCoO2 and PdCoO2. Physical Review Letters 2010, 104(17): 176601.

26. Moll PJW, Kushwaha P, Nandi N, Schmidt B, Mackenzie AP. Evidence for hydrodynamic electron flow in PdCoO2. Science 2016, 351(6277): 1061-1064.

27. Carcia PF, Shannon RD, Bierstedt PE, Flippen RB. O2 Electrocatalysis on Thin-Film Metallic Oxide Electrodes with the Delafossite Structure. Journal of the Electrochemical Society 1980, 127(9): 1974-1978.

28. Hinogami R, Toyoda K, Aizawa M, Kawasaki T, Gyoten H. Copper Delafossite Anode for Water Electrolysis. Ecs Transactions 2013, 58(2): 27-31.

29. Hinogami R, Toyoda K, Aizawa M, Yoshii S, Kawasaki T, Gyoten H. Active copper delafossite anode for oxygen evolution reaction. Electrochemistry Communications 2013, 35: 142-145.

30. Toyoda K, Hinogami R, Miyata N, Aizawa M. Calculated Descriptors of Catalytic Activity for Water Electrolysis Anode: Application to Delafossite Oxides. The Journal of Physical Chemistry C 2015, 119(12): 6495-6501. 
31. Gu J, Yan Y, Krizan JW, Gibson QD, Detweiler ZM, Cava RJ, et al. p-Type CuRhO2 as a SelfHealing Photoelectrode for Water Reduction under Visible Light. Journal of the American Chemical Society 2014, 136(3): 830-833.

32. Prévot MS, Jeanbourquin XA, Bourée WS, Abdi F, Friedrich D, van de Krol R, et al. Evaluating Charge Carrier Transport and Surface States in CuFeO2 Photocathodes. Chemistry of Materials 2017, 29(11): 4952-4962.

33. Varga A, Samu GF, Janáky C. Rapid synthesis of interconnected CuCrO2 nanostructures: A promising electrode material for photoelectrochemical fuel generation. Electrochimica Acta 2018, 272: 22-32.

34. Ahmed J, Mao Y. Delafossite CuAlO2 Nanoparticles with Electrocatalytic Activity toward Oxygen and Hydrogen Evolution Reactions. Nanomaterials for Sustainable Energy, vol. 1213. American Chemical Society, 2015, pp 57-72.

35. Díaz-García AK, Lana-Villarreal T, Gómez R. Sol-gel copper chromium delafossite thin films as stable oxide photocathodes for water splitting. Journal of Materials Chemistry $A$ 2015, 3(39): 19683-19687.

36. Park JE, Hu Y, Krizan JW, Gibson QD, Tayvah UT, Selloni A, et al. Stable Hydrogen Evolution from an AgRhO2 Photocathode under Visible Light. Chemistry of Materials 2018, 30(8): 2574-2582.

37. Oswald H-R, Kuhn P, Reller A. Bimetallic phases from reduction of delafossite-type oxides in hydrogen. Solid State lonics 1989, 32-33: 528-536.

38. Duncan $\mathrm{H}$, Lasia A. Separation of hydrogen adsorption and absorption on Pd thin films. Electrochimica Acta 2008, 53(23): 6845-6850.

39. Tanaka M, Hasegawa M, Takei H. Crystal growth of PdCoO2, PtCoO2 and their solidsolution with delafossite structure. Journal of Crystal Growth 1997, 173(3): 440-445.

40. Takatsu H, Yonezawa S, Mouri S, Nakatsuji S, Tanaka K, Maeno Y. Roles of HighFrequency Optical Phonons in the Physical Properties of the Conductive Delafossite PdCoO2. Journal of the Physical Society of Japan 2007, 76(10): 104701. 
41. Kikugawa N, Goswami P, Kiswandhi A, Choi ES, Graf D, Baumbach RE, et al. Interplanar coupling-dependent magnetoresistivity in high-purity layered metals. Nature Communications 2016, 7: 10903.

42. Morales-Guio CG, Stern L-A, Hu X. Nanostructured hydrotreating catalysts for electrochemical hydrogen evolution. Chemical Society Reviews 2014, 43(18): 6555-6569.

43. McCrory CCL, Jung S, Ferrer IM, Chatman SM, Peters JC, Jaramillo TF. Benchmarking Hydrogen Evolving Reaction and Oxygen Evolving Reaction Electrocatalysts for Solar Water Splitting Devices. Journal of the American Chemical Society 2015, 137(13): 43474357.

44. Cherevko S, Geiger S, Kasian O, Kulyk N, Grote J-P, Savan A, et al. Oxygen and hydrogen evolution reactions on $\mathrm{Ru}, \mathrm{RuO} 2, \mathrm{Ir}$, and IrO2 thin film electrodes in acidic and alkaline electrolytes: A comparative study on activity and stability. Catalysis Today 2016, 262: 170-180.

45. Awaludin Z, Safuan M, Okajima T, Ohsaka T. Investigating the physical and electrochemical effects of cathodic polarization treatment on TaOx. Journal of Materials Chemistry A 2015, 3(32): 16791-16800.

46. Mario C, C. TA, Sergio T. Thermally prepared Ti/RhOx electrodes: II H2 evolution in acid solution. Hemijska industrija 2002, 56(6).

47. Shinagawa T, Garcia-Esparza AT, Takanabe K. Insight on Tafel slopes from a microkinetic analysis of aqueous electrocatalysis for energy conversion. Scientific Reports 2015, 5: 13801.

48. Maoka T, Enyo M. Overpotential decay transients and the reaction mechanism on the Pd-H2 electrode. Surface Technology 1979, 8(5): 441-450.

49. Pentland N, Bockris JO, Sheldon E. Hydrogen Evolution Reaction on Copper, Gold, Molybdenum, Palladium, Rhodium, and Iron - Mechanism and Measurement Technique under High Purity Conditions. Journal of the Electrochemical Society 1957, 104(3): 182194.

50. Searson PC. Hydrogen evolution and entry in palladium at high current density. Acta Metallurgica et Materialia 1991, 39(11): 2519-2525. 
51. Elam M, Conway BE. Sorption Behavior of the Overpotential-Deposited H Species in the Cathodic H 2 Evolution Reaction at Pd and Pt-Pd Electroplated Composite Electrodes. Journal of The Electrochemical Society 1988, 135(7): 1678-1685.

52. Jana R, Bhim A, Bothra P, Pati SK, Peter SC. Electrochemical Dealloying of PdCu3 Nanoparticles to Achieve Pt-like Activity for the Hydrogen Evolution Reaction. ChemSusChem 2016, 9(20): 2922-2927.

53. Greeley J, Nørskov JK, Kibler LA, El-Aziz AM, Kolb DM. Hydrogen Evolution Over Bimetallic Systems: Understanding the Trends. ChemPhysChem 2006, 7(5): 1032-1035.

54. Lin D, Lasia A. Electrochemical impedance study of the kinetics of hydrogen evolution at a rough palladium electrode in acidic solution. Journal of Electroanalytical Chemistry 2017, 785: 190-195.

55. Hollemann AF, Wiberg N. Lehrbuch der Anorganischen Chemie, 102 edn. Walter de Gruyter: Berlin, 2007.

56. Wagner S, Uchida H, Burlaka V, Vlach M, Vlcek M, Lukac F, et al. Achieving coherent phase transition in palladium-hydrogen thin films. Scripta Materialia 2011, 64(10): 978981.

57. Zhang S, Scheu C. Evaluation of EELS spectrum imaging data by spectral components and factors from multivariate analysis. Microscopy 2018, 67(suppl_1): i133-i141.

58. Arblaster JW. Crystallographic Properties of Palladium Assessment of properties from absolute zero to the melting point. Platin Met Rev 2012, 56(3): 181-189.

59. Kuo C-H, Lamontagne LK, Brodsky CN, Chou L-Y, Zhuang J, Sneed BT, et al. The Effect of Lattice Strain on the Catalytic Properties of Pd Nanocrystals. ChemSusChem 2013, 6(10): 1993-2000.

60. Kuhrt C, Anton R. On the origin of a lattice expansion in palladium and Pd?Au vapour deposits on various substrates. Thin Solid Films 1991, 198(1): 301-315. 
61. Teranishi T, Miyake M. Size Control of Palladium Nanoparticles and Their Crystal Structures. Chemistry of Materials 1998, 10(2): 594-600.

62. Dafft EG, Bohnenkamp K, Engell HJ. Investigations of the Electrochemical Reduction of Hydrogen lons on Palladium Electrodes. Zeitschrift für Physikalische Chemie; 1977. p. 33.

63. Hammer B, Norskov JK. Why gold is the noblest of all the metals. Nature 1995, 376: 238.

64. Nilsson A, Pettersson LGM, Hammer B, Bligaard T, Christensen CH, Nørskov JK. The electronic structure effect in heterogeneous catalysis. Catalysis Letters 2005, 100(3): 111-114.

65. Gorzkowski MT, Lewera A. Probing the Limits of d-Band Center Theory: Electronic and Electrocatalytic Properties of Pd-Shell-Pt-Core Nanoparticles. The Journal of Physical Chemistry C 2015, 119(32): 18389-18395.

66. Hakamada M, Nakano H, Furukawa T, Takahashi M, Mabuchi M. Hydrogen Storage Properties of Nanoporous Palladium Fabricated by Dealloying. The Journal of Physical Chemistry C 2010, 114(2): 868-873.

67. Zhou H-B, Jin S, Zhang Y, Lu G-H, Liu F. Anisotropic Strain Enhanced Hydrogen Solubility in bcc Metals: The Independence on the Sign of Strain. Physical Review Letters 2012, 109(13): 135502.

68. Yang Y, Kumar KS. Elastic strain effects on the catalytic response of Pt and Pd thin films deposited on Pd-Zr metallic glass. Journal of Materials Research 2017, 32(14): 26902699.

69. Wagner S, Pundt A. Quasi-thermodynamic model on hydride formation in palladiumhydrogen thin films: Impact of elastic and microstructural constraints. International Journal of Hydrogen Energy 2016, 41(4): 2727-2738.

70. Baldi A, Narayan TC, Koh AL, Dionne JA. In situ detection of hydrogen-induced phase transitions in individual palladium nanocrystals. Nature Materials 2014, 13: 1143. 
71. Ulvestad A, Welland MJ, Collins SSE, Harder R, Maxey E, Wingert J, et al. Avalanching strain dynamics during the hydriding phase transformation in individual palladium nanoparticles. Nature Communications 2015, 6: 10092.

72. Akiba H, Kofu M, Kobayashi H, Kitagawa H, Ikeda K, Otomo T, et al. Nanometer-Size Effect on Hydrogen Sites in Palladium Lattice. Journal of the American Chemical Society 2016, 138(32): 10238-10243. 